\title{
NONINVASIVE ASSESSMENT OF LARYNGEAL PHONATION FUNCTION USING COLOR DOPPLER ULTRASOUND IMAGING
}

\author{
Tzu-Yu Hsiao, ${ }^{*}$ Chung-Li Wang, ${ }^{\dagger}$ Chiung-Nien Chen, ${ }^{\dagger}$ Fon-Jou Hsieh ${ }^{\dagger}$ and \\ YIO-Wha SHAU \\ Departments of *Otolaryngology and ${ }^{\dagger}$ Diagnostic Ultrasound, National Taiwan University Hospital, Taipei, Taiwan; \\ and ${ }^{\ddagger}$ Institute of Applied Mechanics, National Taiwan University, Taipei, Taiwan
}

(Received 2 October 2000; in final form 10 April 2001)

\begin{abstract}
Color Doppler imaging (CDI) was used to identify the morphology of vocal folds (VF) and to quantify the tissue horizontal displacement velocity (HDV) in the vibrating portion of VF. Mucosal HDV that gives an estimate of the stiffness of the VF cover is very important clinically. The VF and its cover were shown to be very hypoechoic and not adequately visible in B-scan image. However, in this study, we found that the structure of the true VF, especially the mucosa and the superficial layer of the lamina propria, the so-called "cover," can be easily identified and evaluated using CDI. The mean VF displacement velocity was measured by decoding the pseudocolor codes of the ultrasound (US) image at the vibrating sites. The mucosal mean HDV obtained from 10 normal men of age $34 \pm 8$ years phonating at their most comfortable pitch and intensity $(98 \pm 12 \mathrm{~Hz}, 55-65 \mathrm{~dB})$ was about $68 \pm 10 \mathrm{~cm} / \mathrm{s}$, which agreed reasonably with the literature. Therefore, the CDI could be used as a potential tool for assessing the phonation function in the larynx nonintrusively. (E-mail: ywshau@spring.iam.ntu.edu.tw) @ 2001 World Federation for Ultrasound in Medicine \& Biology.
\end{abstract}

Key Words: Color Doppler ultrasound image, Vocal fold, Phonation function, Mucosal displacement velocity.

\section{INTRODUCTION}

Clinical application of ultrasound (US) imaging has not been widely accepted in laryngology (Miller and Nemechek 1998). The true vocal folds (VF) and the vocal muscles appeared as hypoechoic structures that could not be adequately visualized in US scanning (Raghavendra et al. 1987). Morphologically, the use of B-mode US is not superior to other imaging techniques, such as CT or MRI in visualizing laryngeal structures, especially the true VF (Miller and Nemechek 1998). Therefore, the US studies of phonation function have been limited (Hertz et al. 1970; Schindler et al. 1990).

The vibration of VF periodically interrupts the glottal airflow and forms the acoustic signal that is perceived as the voice (Hirano 1975). Due to its unique multilayered structure, the VF can be divided into the "cover" and the "body" (Hirano 1974). The cover includes mucosa and the superficial layer of lamina propria, called

Address correspondence to: Yio-Wha Shau, Ph.D., Associate Professor, Biophysics and Gasdynamics Lab., Institute of Applied Mechanics, National Taiwan University, 1, Roosevelt Rd. Sec.4, Taipei, 106, Taiwan. E-mail: ywshau@ spring.iam.ntu.edu.tw the Reinke's space. The body consists of vocal ligament and vocal muscle. Functionally, the vibration of VF is confined mainly to the cover and the mucosal wave is propagated vertically from the lower to the upper margin of the VF (Titze 1988; Titze et al. 1993). The vertical mucosal traveling wave is the summation of sequential horizontal tissue displacement waves from the infraglottic to the supraglottic extent of the VF structure (Fig. 1). Both the vertical and the horizontal waves can be approximated by sine waves.

The stiffness of the VF cover has been found to play an important role in the formation of the mucosal wave and the laryngeal phonation function. Several in vivo methods have been designed to measure human VF stiffness or elastic modulus directly by tagging the VF cover (Tran et al. 1993; Tanaka and Hirano 1990). Some authors instead measure the displacement velocity, which is the horizontal component of the mucosal wave velocity, using a laryngoscope to estimate the stiffness indirectly (Nasri et al. 1994; Hanson et al. 1995). However, these methods were either invasive or inconvenient, and could not reveal the underlying VF structural properties. Therefore, their application in routine clinical examination was 


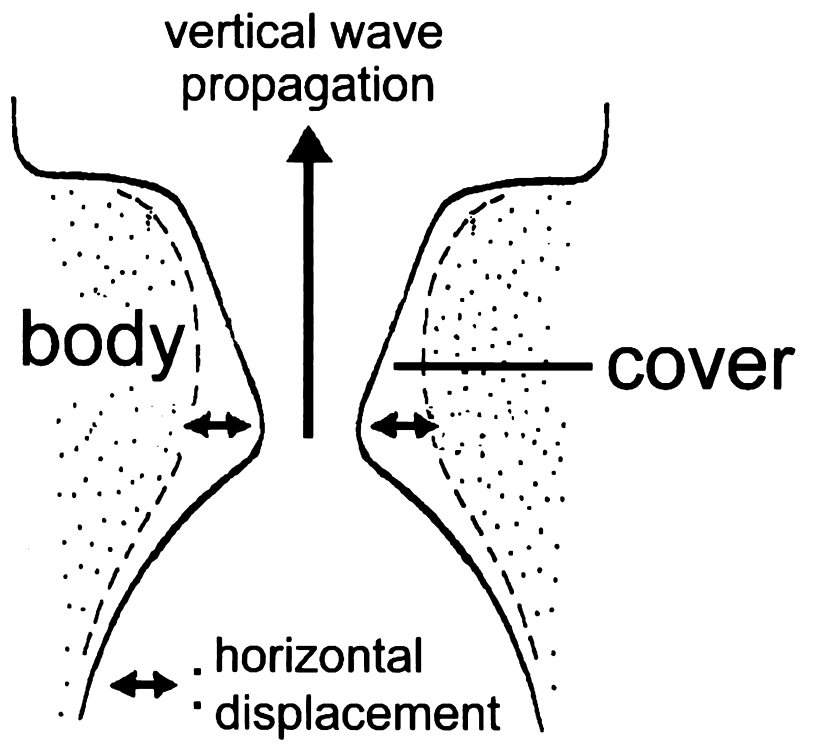

Fig. 1. Schematic diagram for showing the cover-body and vertical-horizontal wave of vocal fold vibration.

somewhat restricted. It is apparent from previous studies that the mucosal vertical wave velocity (VWV) is positively related to the stiffness of the VF (Titze 1988) and is linearly proportional to the tissue horizontal displacement velocity (HDV) (Berke 1992). Therefore, the measurement of HDV can be used to estimate the VWV and then to predict the VF stiffness (Nasri et al. 1994).

The aim of this study was to find an in vivo, noninvasive method to quantify the human laryngeal structural function with minimum disturbance using a commercially available US system. Color duplex sonography has been widely applied in clinics in recent years. The system uses Doppler effect to reveal the velocity of blood flow or tissue motion toward or away from the scan head. A pseudocolor velocity map is placed on top of grey-scale images to show the mean velocity locally (Ferrara and DeAngelis 1997; Hoskins and McDicken 1997). By using color Doppler imaging, the mean velocity of tissue displacement in the VF cover area can be measured. In this study, we first verified the morphologic appearance of VF in the B-mode image. Then, we applied the color mode to identify the VF vibration patterns and measure the corresponding mucosal HDV.

\section{MATERIALS AND METHODS}

The study was performed with a commercially available, high-resolution US scanner (HDI-5000, ATL, Bothell, WA) with a 5-12-MHz linear-array transducer (L12-5 $38 \mathrm{~mm}$, ATL). To visualize the VF structure at a depth of up to $4 \mathrm{~cm}$, the frame rate was typically 15-24

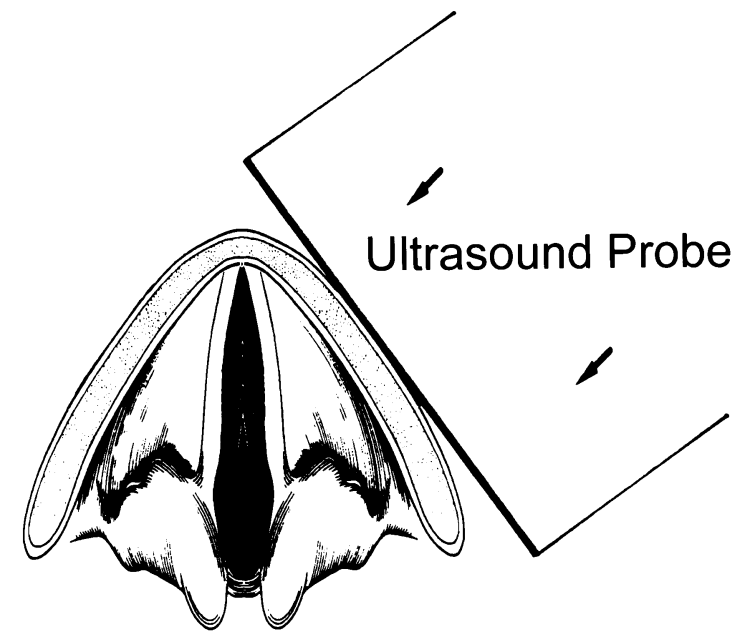

(a)

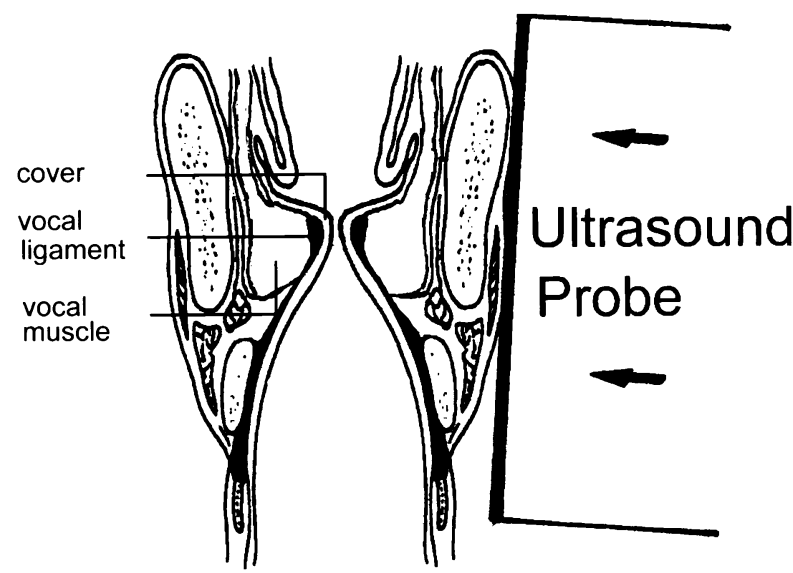

(b)

Fig. 2. Schematic diagrams of the US transducer's position and direction. (a) Horizontal view of the vocal folds; (b) coronal view of the vocal folds.

$\mathrm{Hz}$ in B-mode. In the color mode, the pulse repetition rate was $10,000 \mathrm{~Hz}$, and the measuring velocity range was set at 0 to $128.3 \mathrm{~cm} / \mathrm{s}$ with maximum scale and baseline offset that resulted in a frame rate of about $7 \mathrm{~Hz}$. The viewing window of depth $4.8 \mathrm{~cm}$ was high enough to detect the vibratory part of the VF in most of the cases. The US scanner was placed both horizontally and coronally (Fig. 2a and b, respectively).

In B-mode scanning of the VF, the US transducer was placed on the anterolateral aspect of the neck. For the horizontal view, US scanner was placed in the middle of the thyroid lamina on one side because the true VF lay on the plane at this level. The interface between the true 
VF mucosa and the air column in the larynx can be identified as a hyperechoic line. For the coronal view, the scanner was placed vertically at the anterior junction of $\mathrm{VF}$, which is about one fourth of the thyroid cartilage lamina. The midportion in anteroposterior dimension of VF was about the location where VF vibrated at the maximal excursion (Isshiki 1989).

Three women receiving laryngomicrosurgery for the removal of unilateral VF polyps were examined to verify the morphology of the true VF in this ultrasonic study. Under general anesthesia through endotracheal intubation, a metallic instrument used regularly in the microsurgery was placed at the lumen side of the normal VF as US landmark (Fig. 3). The microsurgical instrument was moved back and forth to show the true VF in the B-scan images.

Ten men, aged $34 \pm 8$ years (range $24-50$ years) without any history of voice disorder were involved in the mucosal HDV study. During US scanning, they were in sitting position and were asked to utter a sustained vowel "aa" that corresponded to the individual's most comfortable pitch. The fundamental frequency of the voice was recorded on tape and measured on site with a digital oscilloscope (TDS-224, Tektronix, Wilsonville, OR) equipped with a fast Fourier transform (FFT) module (TDS2MM). The fundamental frequency measured for the utterances "aa" ranged from $98 \pm 12 \mathrm{~Hz}$. For the HDV measurements at a comfortable intensity, the subjects were asked to give a sustained vowel with the mean sound pressure level (SPL) kept at about 55-65 dB that was measured at about $20 \mathrm{~cm}$ away from the mouth. The US B-scan was carried out first to locate the true VF, and then the machine was shifted to color mode for the tissue motion measurements. At least 20 frames were recorded in the sustained vowel uttering (Fig. 4), and at least 5 measurements were taken for analysis in each subject. Because the direction of HDV is parallel to the US scanning line in the coronal section, one can obtain the HDV directly from the color of the pixels at the area of true VF cover.

The RGB (red, green and blue) codes given by the velocity color-bar of CDI were first analyzed. The red code value $(R)$ was found ranging from 57 to 239 with a second order relation to the velocity (Fig. 5). After decoding the red color $(R)$ of the CDI pixels in the vibrating portion of $\mathrm{VF}$, the movement velocities $(V)$ were calculated by fitting $R$-value on the regression line:

$$
V=0.0017 R^{2}+0.2082 R-19.263 .
$$

The HDV value can be determined from the maximal velocity in the vibrating portion of VF that was averaged over the 20 frames recorded.

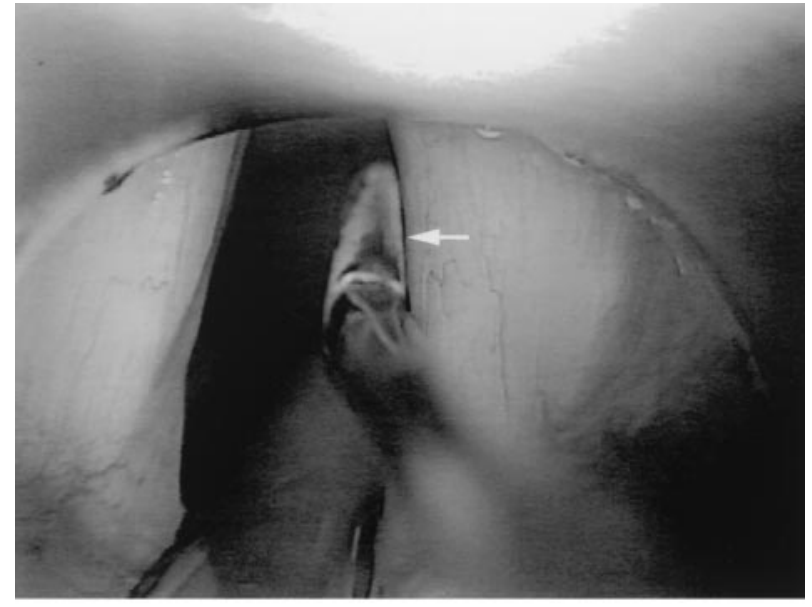

(a)

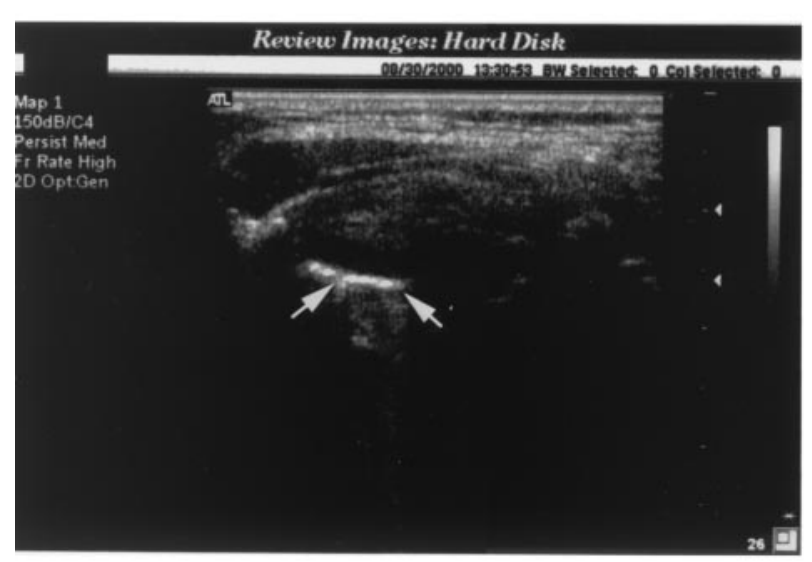

(b)

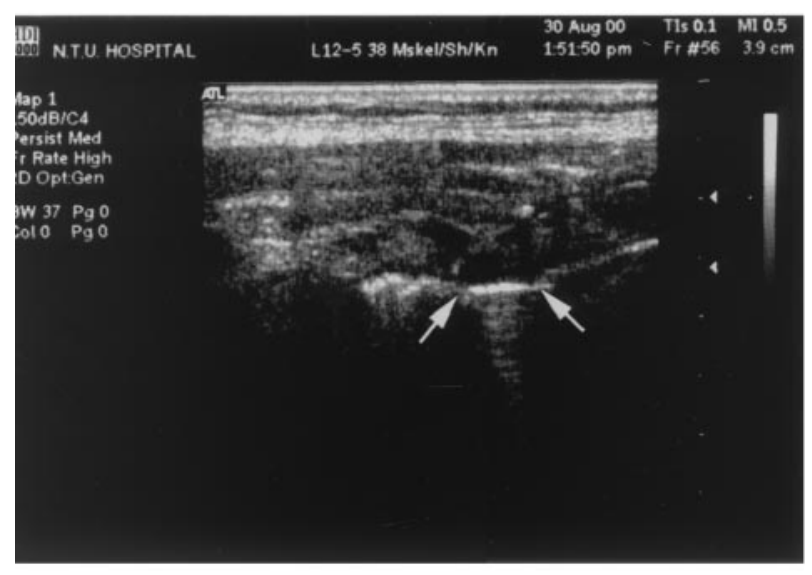

(c)

Fig. 3. (a) The identification of the structure of the vocal fold cover was performed in the B-scan by using the metallic instrument contacting the right vocal fold free margin as a contrast (arrow). (b) In the horizontal section, the cover of the vocal fold was a hypoechoic band. The structure was verified by the metallic comet-tail shadow (arrow). (c) In the coronal section, the cover was identified as a hypoechoic area and it was also verified by the metallic shadow (arrow). 


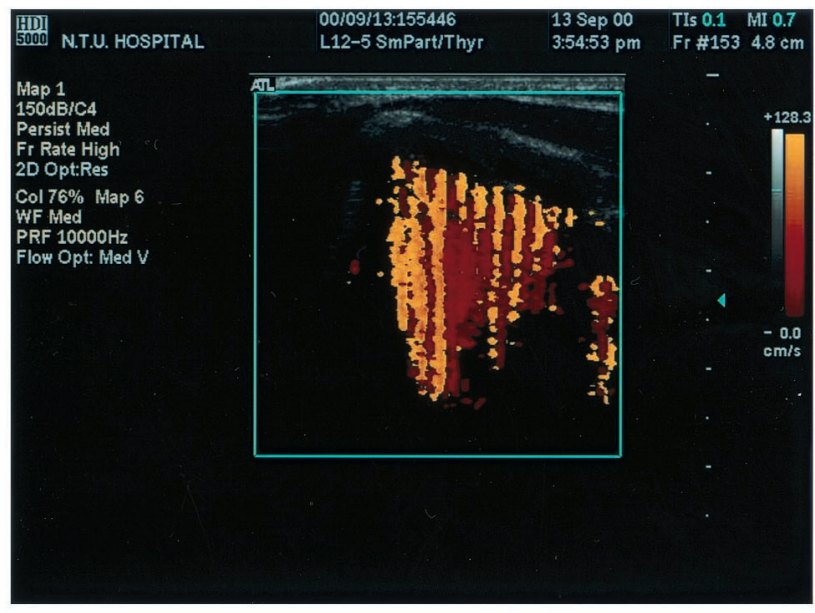

(a)

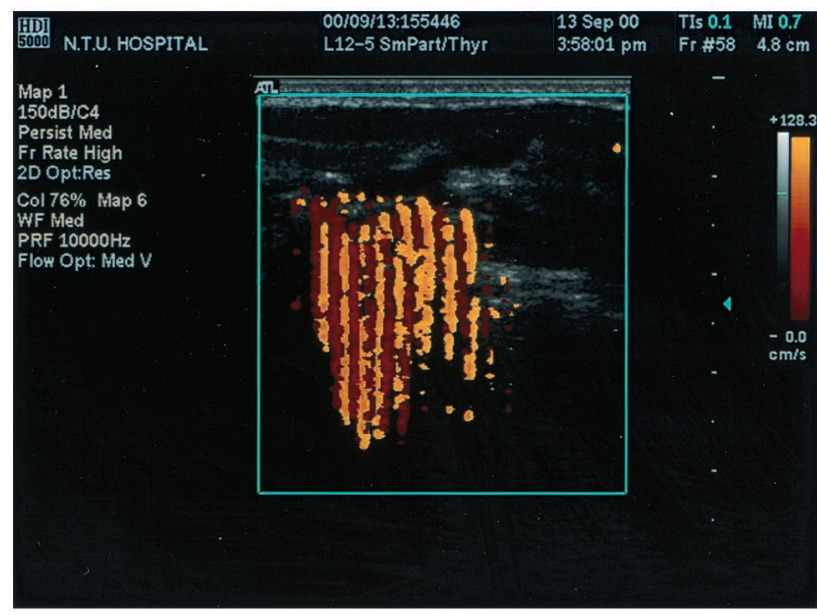

(b)

Fig. 4. Color Doppler images of vocal fold vibration in (a) horizontal plane and in (b) coronal plane. The vibratory motion of the mucosa-air column interface generates the color artifact patterns underneath.

\section{RESULTS}

Under general anesthesia in laryngomicrosurgery, the VFs were in the lateral position. By attaching a metal instrument to the VF free margin, the cover of VF was identified as a hypoechoic band medical to the vocal ligament and vocal muscle in the B-scan image. This structure was easily verified by the US comet-tail shadow that is commonly seen in US images for metallic objects (Fig. 3b and c).

In the real-time B-scan of $\mathrm{VF}$, the adduction and abduction motion of $\mathrm{VF}$ reflected on the movement of arytenoid cartilages and vocal ligaments. Again, the cover of VF was not clearly visualized in the US images. During the utterance of the sustained vowel, a slow wave-like movement was observed in the cover area. By

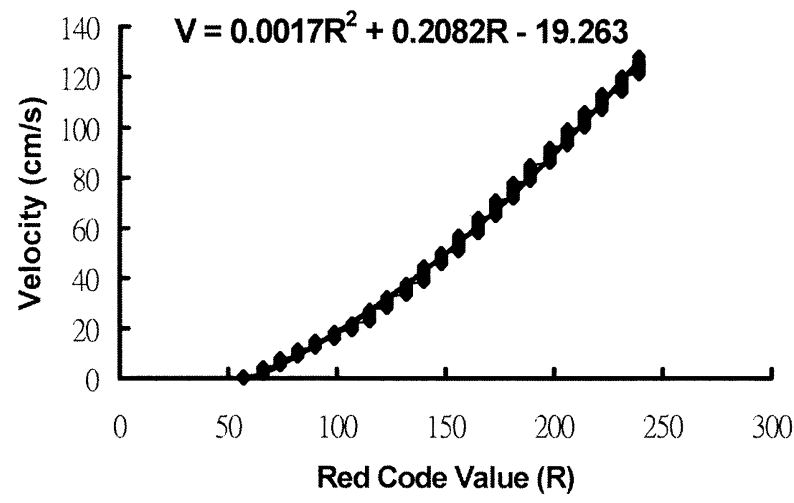

Fig. 5. Calibration curve for the vocal fold displacement velocity $(V)$ vs. the red code $(R)$ of the color-map in CDI. The horizontal displacement velocity (HDV) can be measured, based on the $R$ value.

applying Doppler spectral analysis to the motion in this area, the pitch of the spectral acoustic signals was perceptually indistinguishable from the uttering vowel.

As soon as the US system shifted to the color mode, there were very strong color signals in the vibratory area of VF during phonation (Fig. 4a and b). The pseudocolor signals appeared not only in the cover area of VF, but also in the adjacent region where the vibration waves spread out. When the voice ceased, either in Valsalva status or in quiet respiration position, the color signals disappeared immediately. The Doppler signals apparently came from the fluctuations of VF cover toward or away from the US scan head. After decoding the color signals, the HDV was about $68 \pm 10 \mathrm{~cm} / \mathrm{s}$ in the $10 \mathrm{men}$ phonating at their most comfortable pitch and intensity.

\section{DISCUSSION}

Phonation is one of the major functions in the larynx. This process involves the vibration of VF that modulates the glottal airflow into voice (Hirano 1975). The VF is a multilayered structure anatomically, and can be divided into a cover and a body functionally. The cover is much more pliable than the body and is the main vibratory portion of the VF (Saito et al. 1983). For an elastic medium, the travelling were velocity (TWV) is correlated directly to its stiffness or elastic modulus and is inversely proportional to the mass density (Berke 1992).

$$
T W V=\sqrt{\frac{\Delta Y}{\rho}}
$$

where $\Delta Y$ is the change in elastic modulus and $\rho$ is the mass density. Therefore, the stiffness of the cover is the key factor that affects the formation of the vibratory mucosal 
wave. Although the propagation velocity of the mucosal wave has been recognized to be a significant element in the diagnosis and treatment of laryngeal disorder, it is often neglected in laryngology due to the lack of nonintrusive diagnostic technique. Moreover, it is extremely difficult to measure VF stiffness in awake humans, especially during their normal phonation. So far, in vivo measurement of VF stiffness or elasticity can only be made via invasive methods (Tran et al. 1993; Tanaka and Hirano 1990).

The human VF vibrates in complex three-dimensional (3-D) wave patterns. As the VF vibrates, the vibratory tissue oscillates separately on its horizontal plane, and the vertical mucosal wave travels from the infraglottic to the supraglottic margin of the VF structure and spreads to the adjacent tissues. Based on Hirano's body-cover theory (Hirano 1974), the stiffness of the VF was contributed by both the extrinsic longitudinal tension on the cover and the internal stiffness of the underlying body. In vivo canine experimental evidence showed that the mucosal muscular tension, VF stiffness and VWV were well correlated (Titze et al. 1993), and the VWV ranged from 0.9 to $1.6 \mathrm{~m} / \mathrm{s}$ and was linearly related to HDV (Sloan et al. 1993).

Nasri et al. (1994) developed a videostroboscopic system to visualize the upper edge of the VF in the canine larynx without touching the VF. The levels of phonation were controlled by stimulating the dog's superior and recurrent laryngeal nerves. To calculate the HDV, the VF surface displacement, given by the two video images taken at the most open and the most closed positions, was divided by the time interval. Assuming that the dog's VF mass density was $1 \mathrm{~g} / \mathrm{cm}^{3}$, the HDV calculated was about 0.32 to $1.0 \mathrm{~m} / \mathrm{s}$ and the VWV ranged from 0.76 to $2.2 \mathrm{~m} / \mathrm{s}$. The HDV was shown to correlate linearly with mucosal traveling wave velocity. Therefore, the HDV may be used to reflect the voice disorder associated with the change of mucosal stiffness or mass density.

For VF during phonation, the vibrating frequency typically ranges from about 70 to $500 \mathrm{~Hz}$ in men and 150 to $1000 \mathrm{~Hz}$ in women (Hirano 1981); the frame rate of the commercially available US systems is still not able to catch up with the VF movement in real time. However, there have been a few reports that used US imaging to demonstrate the morphology, movement and vibration of the VF. In the study by Raghavendra et al. (1987), the vocal muscles were hypoechoic structures and the vocal ligaments were echogenic bands that appeared as free margins of the VF. In their study, the adduction and abduction movements of the bilateral vocal ligaments and the arytenoid cartilages were identified during normal breathing using US. However, the true VF could be visualized in only two thirds of the women and in less than half of the men whose mean age was about 25 . The cover of VF was not visible in the B-mode imaging, either. Miles (1989) also demonstrated the VF movement by showing US images of vocal ligament in B-mode and M-mode scanning. Apparently, the VF cover cannot be easily differentiated from its surroundings in the B-mode morphological image. In the current study, we placed a metallic microsurgical instrument contacting the lumen side of the VF as the US contrast to appreciate the cover structure. As our results revealed, the cover was almost nonechogenic and could not clearly visualized under normal circumstances.

The VF vibration is confined largely to the cover, but the VF cover is not adequately visible in the B-mode image. It is, thus, difficult to study the VF vibration using conventional US imaging. Moreover, for the vibration frequency as high as several hundred $\mathrm{Hz}$, to quantify the VF uttering vibration with US is generally not acceptable in the previous reports. Hertz et al. (1970) traced the continuous movement of vocal ligament during VF vibration with an M-mode-like design, and the echo trace was similar to photoglottography. Schindler et al. (1990) used a Doppler US flowmeter to detect the VF vibration velocity in terms of frequency shift; the Doppler frequency was found to vary from a few hundred $\mathrm{Hz}$ to $8000 \mathrm{~Hz}$ in the range of audible frequency. In our study, the frame rate of B-mode scan was about $15-24 \mathrm{~Hz}$, depending on the 2-D scanning line density. With appropriate focusing, the real-time US display of VF vibration showed a slow wave-like motion at the cover area. This motion observed in B-mode was actually the "strobe motion" of the VF movement during phonation.

In the present study, CDI was used to visualize the structural characteristics of the larynx, and it provided an excellent tool to visualize the tissue movements over a large area (Ferrara and DeAngelis 1997; Hoskins and McDicken 1997). Mostly this technique was used to study the blood flows of vessels over a certain region. However, McDicken et al. (1992) applied CDI to investigate the myocardial contraction velocity. Using a similar idea, elastographic imaging is being developed and aimed to differentiate the various biomechanical attributes of tissues (Ophir et al. 2000).

Baken and Orlinkoff (2000) conducted an extensive review of the clinical measurements of phonation function associated with the voice disorder. In the literature, Ooi et al. (1995) applied the CDI qualitatively to identify vocal cord palsy based on the asymmetry of image patterns. Friedman (1997) applied B-mode US to the diagnosis of vocal cord paralysis in infants and children. In this study, we used CDI to estimate the VF stiffness via the measurement of HDV of the VF during phonation. The vibratory motion of the mucosa-air column interface generated a strong colored US artefact at the cover area of the VF during utterance. The color signals were aggregated in the cover and the adjacent area, where the 
vibratory wave spread. The color signals appeared only in the phonation period or lightly during breathing. After the phonation ceased, the color signals disappeared. By applying the Doppler spectral analysis to the US echo in the cover area, the pitch of the spectral audio output was perceptually indistinguishable from the uttering vowel. Therefore, we had enough evidence to confirm that the color artefact resulted from the vibration of the VF.

Because the mucosal cover moved mediolaterally in the direction toward and away from the scan head in the VF coronal view, the HDV of the cover was retrieved from the corresponding CDI based on the velocity color map. The HDV followed approximately a sine curve, in which the peak velocity appeared at the neutral position and zero velocity appeared at its maximal excursion. It should be noted that the magnitude of velocity displayed in CDI is an averaged speed of the moving objects. The HDV was defined as the highest velocity obtained in the area of mucosal cover that averaged for the 20 frames of images taken continuously.

Our results of HDV obtained in the 10 normal men uttering a sustained vowel in their most comfortable pitch and intensity fall in the same range as the data reported in the literature (Titze et al. 1993; Nasri et al. 1994). We also observed that the magnitude of HDV increased, not only with the pitch of the phonation, but also with the intensity of the voice (i.e., the sound pressure level). How this relationship deviates from the sinusoidal wave linear theory requires further study. The present method is intended to give a quantitative estimation of the mechanical properties of the VF and facilitates the potential for further research in laryngeal function.

To our best knowledge, this is the first time that the HDV along the VF is reported in human subjects with normal active phonation using our US system. Using CDI in the measurement of HDV of the VF cover has the advantages over laryngostroboscopy that the VF vibration can be monitored continuously during phonation, with no irritating instruments in the mouth and with no interference to the articulatory movements of the tongue. It can provide a simple, absolutely noninvasive and painless procedure in routine laryngeal functional examination. Moreover, the structural bounding of the VF to its surrounding tissues, in other words, the global nature of VF stress wave propagation, can also be observed.

\section{SUMMARY}

In the literature, the characteristics of the mucosal wave have been shown to correlate directly with the mechanical properties of the VF. The present study indicates that the measurement of HDV using the CDI is feasible and may become a promising technique for assessing the active phonation function of the larynx in humans.
Acknowledgment-This research was supported by Grant NSC 892314-B-002-441.

\section{REFERENCES}

Baken RJ, Orlinkoff RF. Clinical measurement of speech and voice. 2nd ed. Thomson Learning. San Diego: Singular Publishing, 2000.

Berke GS. Intraoperative measurement of the elastic modulus of the vocal fold. Part 1. Device development. Laryngoscope 1992;102: $770-778$.

Ferrara K, DeAngelis G. Color flow mapping. Ultrasound Med Biol 1997;23:321-345.

Friedman EM. Role of ultrasound in the assessment of vocal cord function in infants and children. Ann Otol Rhinol Laryngol 1997; 106:199-209.

Hanson DG, Jiang J, D’Agostino M, Herzon G. Clinical measurement of mucosal wave velocity using simultaneous photoglottography and laryngostroboscopy. Ann Otol Rhinol Laryngol 1995;104:340349.

Hertz CH, Lindstrom K, Sonesson B. Ultrasonic recording of the vibrating vocal folds. Acta Otolaryng 1970;69:223-230.

Hirano M. Morphological structure of the vocal cord as a vibrator and its variations. Folia Phoniatr 1974;26:89-94.

Hirano M. Phonosurgery: Basic and clinical investigations. Otologia (Fukuoka) 1975;21(Suppl. 1):239-262.

Hirano M. Clinical examination of voice. Wien-New York: SpringerVerlag, 1981.

Hoskins PR, McDicken WN. Colour ultrasound imaging of blood flow and tissue motion. Br J Radiol 1997;70:878-890.

Isshiki N. Phonosurgery. Theory and practice. Tokyo: Springer-Verlag, 1989.

McDicken WN, Sutherland GR, Moran CM, Gordon LN. Colour Doppler velocity imaging of the myocardium. Ultrasound Med Biol 1992;18:651-654.

Miles KA. Ultrasound demonstration of vocal cord movements. Br J Radiol 1989;62:871-872.

Miller RH, Nemechek AJ. Airway evaluation and imaging. In: Bailey BJ, Pillsbury HC, Driscoll BP, eds. Head and neck surgeryOtolaryngology. 2nd ed. Philadelphia PA: Lippincott-Raven, 1998: 597-608.

Nasri S, Sercarz JA, Berke GS. Noninvasive measurement of traveling wave velocity in the canine larynx. Ann Otol Rhinol Laryngol 1994;103:758-766.

Ooi LL, Chan HS, Soo KC. Color Doppler imaging for vocal cord palsy. Head Neck 1995;17:20-23.

Ophir J, Garra B, Kallel F, et al. Elastographic imaging. Ultrasound Med Biol 2000;26:S23-S29.

Raghavendra BN, Horii SC, Reede DL, et al. Sonographic anatomy of the larynx, with particular reference to the vocal cords. J Ultrasound Med 1987;6:225-230.

Saito S, Fukuda H, Kitahara S, et al. Pellet tracking in the vocal fold while phonating - experimental study using canine larynges with muscle activity. In: Titze IR, Scherer RC, eds. Vocal fold physiology: Biomechanics, acoustics and phonatory control. Denver: Denver Center for the Performing Arts, 1983:169-182.

Schindler O, Gonella ML, Pisani R. Doppler ultrasound examination of the vibration speed of vocal folds. Folia Phoniatr 1990;42:265-272.

Sloan SH, Berke GS, Garratt BR, Kreiman J, Ye M. Determination of vocal fold mucosal wave velocity in an in vivo canine model. Laryngoscope 1993;103:947-953.

Tanaka S, Hirano M. Fiberscopic estimation of vocal fold stiffness in vivo using the sucking method. Arch Otolaryngol Head Neck Surg 1990;116:721-724.

Titze IR. The physics of small-amplitude oscillation of the vocal folds. J Acoust Soc Am 1988;83:1536-1552.

Titze IR, Jiang JJ, Hsiao TY. Measurement of mucosal wave propagation and vertical phase difference in vocal fold vibration. Ann Otol Rhinol Laryngol 1993;102:58-63.

Tran QT, Berke GS, Gerratt BR, Kreiman J. Measurement of Young's modulus in the in vivo human vocal folds. Ann Otol Rhinol Laryngol 1993;102:584-591. 